\title{
Increase in the fructooligosaccharides yield and productivity by solid-state fermentation with Aspergillus japonicus using agro-industrial residues as support and nutrient source
}

\author{
Solange I. Mussatto*, José A. Teixeira \\ IBB - Institute for Biotechnology and Bioengineering, Centre of Biological Engineering, University of Minho, Campus de Gualtar, 4710-057 Braga, Portugal
}

\section{A R T I C L E I N F O}

\section{Article history:}

Received 16 July 2010

Received in revised form

10 September 2010

Accepted 17 September 2010

\section{Keywords:}

Fructooligosaccharides

Solid-state fermentation

Nutrient

Aspergillus japonicus

$\beta$-Fructofuranosidase

\begin{abstract}
A B S T R A C T
Corn cobs, coffee silverskin, and cork oak were used as support and nutrient source during the fructooligosaccharides (FOS) production by Aspergillus japonicus, under solid-state fermentation (SSF) conditions. The objectives of this study consisted in evaluating the possibility of improving the FOS yield and productivity, besides to finding an alternative to reduce the production costs, and add value to these agro-industrial residues. Fermentation assays were performed by using the materials as solid support, supplemented or not with nutrients. For comparison, assays were also performed using a synthetic material as solid support, under the same operational conditions. All the material residues acted as nutrient source for the microorganism, since FOS production occurred when all of them were used without nutrient supplementation, but not when the synthetic material was used. Among the evaluated materials, coffee silverskin gave the most interesting fermentation results, with a FOS production similar in both supplemented and non-supplemented media. The elevated FOS production $(128.7 \mathrm{~g} / \mathrm{l})$ and $\beta$-fructofuranosidase activity $(71.3 \mathrm{U} / \mathrm{ml})$ obtained by using this material suggest SSF of coffee silverskin with A. japonicus as an interesting and promising strategy to synthesize both products at the industrial level.
\end{abstract}

(c) 2010 Elsevier B.V. All rights reserved.

\section{Introduction}

The development of prebiotic products has gained large attention in the last decades, due to the increased consumer desire to improve health through food. Among the new commercially available prebiotic ingredients, the fructooligosaccharides (FOS), fructose oligomers obtained from sucrose, have attracted special attention due to their important functional properties including low caloric value, non-cariogenicity, effects in decreasing the levels of phospholipids, triglycerides, and cholesterol, help in gut absorption of calcium and magnesium, and usefulness to stimulate bifidobacterial growth in the human colon $[1,2]$.

FOS is produced commercially through the enzymatic synthesis from sucrose by microbial enzymes with transfructosylating activity ( $\beta$-fructofuranosidases, EC.3.2.1.26, also designed as fructosyltransferases EC.2.4.1.9). Most of these enzymes have been found in fungi such as Aspergillus, Aureobasidum, and Penicillium [3,4], among of which Aspergillus species have received particular attention and have been cited as good enzyme producers. In

\footnotetext{
* Corresponding author. Tel.: +351 253604 424; fax: +351 253678986. E-mail addresses: solange@deb.uminho.pt, solangemussatto@hotmail.com (S.I. Mussatto).
}

the past few years, several Aspergillus japonicus strains have been reported as potentially adequate for industrial production of FOS and $\beta$-fructofuranosidase $[4,5]$.

The main drawback of the commercial FOS production by transfructosylation is that the yields are normally low (55-60\%) $[2,6]$. Therefore, and due to the increased demand for using these ingredients in food and pharmaceutical products, there is a great interest in the development of a suitable and economically viable biotechnological process for industrial production of FOS that allow obtaining higher yields and productivities. Most investigations about experimental conditions for FOS and $\beta$-fructofuranosidase production are mainly based on submerged fermentation $(\mathrm{SmF})$ experiments. However, solid-state fermentation (SSF) systems appear to be an interesting alternative to obtain higher volumetric productivity and product concentration, with lower capital cost and energy consumption when compared to SmF [7]. SSF requires low water volume and thus has large impact on the economy of the process due to smaller fermenter-size, reduced downstream processing, reduced stirring and lower sterilization costs $[8,9]$. In addition, the use of low cost agricultural and agro-industrial residues as substrates, contributes also to lower capital and operating costs compared to SmF.

The FOS production by SSF systems have been few explored until nowadays. To the best of our knowledge, only one published study 
reported the use of this system for the production of the fructosyltransferase enzyme by Aspergillus oryzae [10]. In the present work, three agro-industrial residues were used in SSF systems for the production of FOS and $\beta$-fructofuranosidase by $A$. japonicus. The residues included corn cobs, coffee silverskin and cork oak, which were previously found as being materials with ability for immobilization of $A$. japonicus during the FOS production by SmF [11]. Fermentation assays were performed to verify the kinetic behavior of FOS and $\beta$-fructofuranosidase production by SSF, and the possibility of using these agro-industrial residues as nutrient source during this process.

\section{Materials and methods}

\subsection{Solid supports}

Three agro-industrial residues (corn cobs, coffee silverskin and cork oak) and one synthetic material (synthetic fiber Scotch Brite, $3 \mathrm{M}$ Spain, SA), were used as solid support during the SSF. Corn cobs and cork oak were obtained from local farmers; coffee silverskin was supplied by NovaDelta-Comércio e Indústria de Cafés S.A. (Campo Maior, Portugal), and the synthetic fiber was purchased in a local market. To be used in the experiments, particles with approximately $3 \times 3 \mathrm{~mm}$ (length $\times$ width) were selected and autoclaved at $121^{\circ} \mathrm{C}$ for $20 \mathrm{~min}$.

\subsection{Microorganism and cultivation conditions}

The strain A.japonicus ATCC 20236 was used in the experiments. The strain was maintained on potato dextrose agar (PDA - Difco) plates at $4{ }^{\circ} \mathrm{C}$, and the spores were maintained mixed with glycerol solution in ultra-freezer at $-80^{\circ} \mathrm{C}$. For the production of spores the strain was grown on PDA medium, at $28-30^{\circ} \mathrm{C}$ for $7-8$ days.

\subsection{Solid-state fermentation (SSF)}

The SSF cultivations were performed in Petri dishes containing approximately $3.0 \mathrm{~g}$ of the previously sterilized materials. For the experiments, the materials were moistened with a $200 \mathrm{~g} / \mathrm{l}$ sucrose solution to attain $70 \%$ moisture content, inoculated with a concentrated spore suspension to give $2 \times 10^{6}$ spores/g material, and statically incubated at $28^{\circ} \mathrm{C}$ during $48 \mathrm{~h}$. Cultivations were performed with the sucrose solution supplemented or not with the following nutrients (g/l): yeast extract (27.5), $\mathrm{NaNO}_{3}(2.0), \mathrm{K}_{2} \mathrm{HPO}_{4}$ (5.0), $\mathrm{MgSO}_{4} \times 7 \mathrm{H}_{2} \mathrm{O}(0.5)$, and $\mathrm{KCl}(0.5)$. Steam sterilization of the media was carried out at $112^{\circ} \mathrm{C}$ for $15 \mathrm{~min}$. The spores' suspension was prepared by scrap down the spores from the PDA plates with a sterilized solution of $1 \mathrm{~g} / 1$ Tween 80 , and counted in a Neubauer chamber.

Samples for analysis were collected at regular intervals. The total content of each Petri dish was collected as a sample and $2 \mathrm{ml}$ of sterilized distilled water was added to facilitate the fermented broth extraction. After the water incorporation to the fermented solid material, the mixture was submitted to a vacuum filtration process to extract the fermented broth, which was subsequently filtered in $0.2 \mu \mathrm{m}$ filters. In the filtered broth, FOS (1-kestose, 1-nystose, and $1-\beta$-fructofuranosyl nystose), residual concentration of other sugars (sucrose, fructose, and glucose), and extracellular enzyme activity were measured. All the results obtained in these analyses were calculated considering only the moisture content present in the material, being thus corrected by the dilution caused during the water addition to extract the fermented broth. All the experiments were conducted in duplicate.
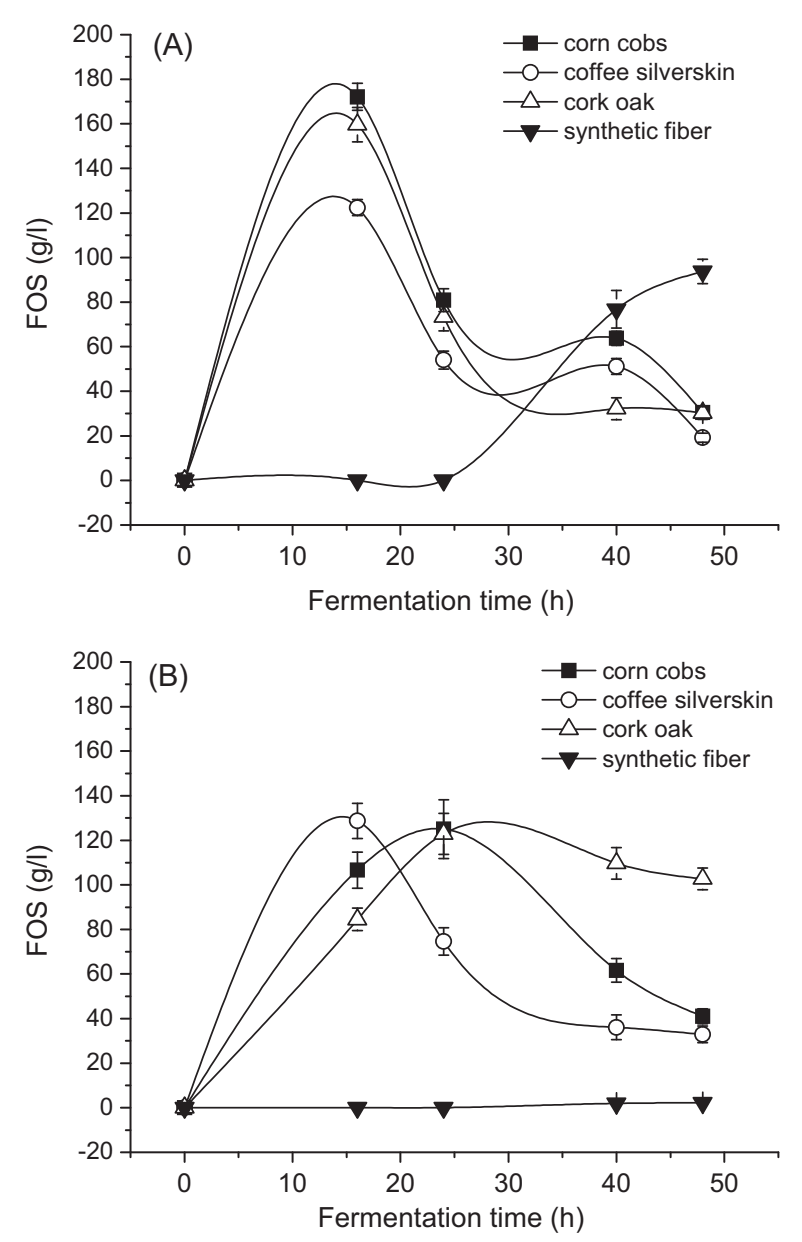

Fig. 1. FOS production by Aspergillus japonicus under solid-state fermentation conditions using different materials as solid support. Assays supplemented (A) or not (B) with nutrients.

\subsection{Analytical methods}

The concentration of sucrose, glucose, fructose, 1-kestose, 1 -nystose, and 1- $\beta$-fructofuranosyl nystose, and the $\beta$ fructofuranosidase (FFase) activity were determined as previously described [11].

\subsection{Fermentative parameters calculation}

The total FOS concentration was calculated by the sum of the concentrations of 1-kestose, 1 -nystose, and 1- $\beta$-fructofuranosyl nystose. The FOS yield $\left(Y_{\mathrm{P} / \mathrm{S}}, \mathrm{g} / \mathrm{g}\right)$ was determined by the ratio between total FOS $(\mathrm{g} / \mathrm{l})$ and consumed sucrose $(\mathrm{g} / \mathrm{l})$. FOS productivity $\left(Q_{P}\right)$ was calculated as the total FOS $(\mathrm{g} / \mathrm{l})$ by fermentation time (h).

\section{Results and discussion}

Fig. 1 shows the FOS production by A. japonicus under SSF conditions using the different materials as solid support. Fig. 1A shows the results obtained for the assays supplemented with nutrients; while Fig. 1B shows the results obtained for the assays where no nutrients were added to the media (the materials were moistened with sucrose solution only). As can be noted, all the material residues used in the experiments acted as nutrient source for the microorganism since FOS production occurred from all of them but not when using the synthetic fiber (Fig. 1B). Among the three tested 
Table 1

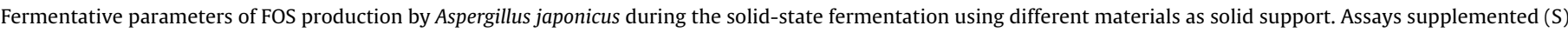
or not supplemented (NS) with nutrients.

\begin{tabular}{|c|c|c|c|c|c|c|}
\hline \multirow[t]{2}{*}{ Solid support material } & \multicolumn{2}{|l|}{$Y_{\mathrm{P} / \mathrm{S}}(\mathrm{g} / \mathrm{g})^{\mathrm{a}}$} & \multicolumn{2}{|l|}{$Q_{P}(g / l h)^{b}$} & \multicolumn{2}{|c|}{ Fermentation time $(\mathrm{h})$} \\
\hline & $\mathrm{S}$ & NS & $\mathrm{S}$ & NS & $\mathrm{S}$ & NS \\
\hline Corn cobs & $1.00 \pm 0.03$ & $0.61 \pm 0.07$ & $10.76 \pm 0.37$ & $5.21 \pm 0.55$ & 16 & 24 \\
\hline Coffee silverskin & $0.96 \pm 0.02$ & $0.70 \pm 0.04$ & $7.64 \pm 0.22$ & $8.05 \pm 0.49$ & 16 & 16 \\
\hline Cork oak & $0.97 \pm 0.07$ & $0.63 \pm 0.03$ & $9.98 \pm 0.48$ & $5.12 \pm 0.21$ & 16 & 24 \\
\hline Synthetic fiber & $0.69 \pm 0.09$ & $0.04 \pm 0.01$ & $1.96 \pm 0.17$ & $0.05 \pm 0.01$ & 48 & 48 \\
\hline
\end{tabular}

a FOS yield factor as the ratio between total FOS produced and consumed sucrose.

b FOS productivity as the ratio between FOS produced per fermentation time.

materials, it was observed a reduction in the FOS production when corn cobs and cork oak were used as nutrient source, when compared to the results obtained from nutrient supplemented media. Such results suggest that these materials contain nutrients that are metabolized by the microorganism, but probably these nutrients are not found in enough amounts to reproduce the results achieved from supplemented media. On the other hand, coffee silverskin gave FOS production results similar in both assays, supplemented or not with nutrients, suggesting that this material is nutritionally richer than cork oak or corn cobs, and that is able to be used as nutrient source for FOS production with no requirement of additional nutrient supplementation.

The fermentative parameter values obtained in these SSF assays are summarized in Table 1. When comparing the FOS production results from supplemented media, it is evident that the residual materials contributed with some additional nutrient source to the microorganism besides those added to the medium, since FOS production was higher than that achieved when using the synthetic fiber as solid support. Corn cobs, particularly, yielded a 2-fold higher FOS production than that achieved using synthetic fiber. On the other hand, with exception of coffee silverskin, FOS production for the other solid supports was strongly affected in the assays without nutrient addition. The almost null production of FOS when using synthetic fiber clearly shows the microorganism necessity for some nutritional source to produce FOS. Part of these requirements were found in corn cobs and cork oak, but the amount present in these materials was not able to provide the same FOS results found in the supplemented assays. Otherwise, coffee silverskin provided the nutrients required to the microorganism reproduce the results achieved from nutrient supplemented medium. In this case, the non-improvement in the FOS production results when the medium was supplemented with nutrients could be explained by the excess of nutritional sources in the medium. Since coffee silverskin contained enough nutrients for the microorganism development, the excess was not utilized by the microorganism, being thus unnecessary for the process. Considering that the FOS production is dependent of the nutrient addition to the fermentation medium, the no necessity of adding nutrients in SSF can be considered a very important economical aspect.

When observing the yield and productivities values shown in Table 1 , it can be noted that similar FOS production yields $\left(Y_{\mathrm{P} / \mathrm{S}}\right)$ were obtained for the non-supplemented media containing the agro-industrial residues. Such yields, correspondent to $61-70 \%$, were higher than the values usually reported for the commercial FOS production (55-60\%) [2,6], and represent an important improvement for the FOS production at industrial level. In addition, the yield values here found are also higher than that $(0.24 \mathrm{~g} / \mathrm{g})$ achieved during the FOS production by $A$. japonicus in a repeated batch fermentation system with 3 consecutive cycles [5], and are similar to that obtained by SmF with immobilized A. japonicus [11] but using a fermentation medium supplemented with nutrients.

Regarding the FOS productivity $\left(Q_{P}\right)$, coffee silverskin promoted the highest value of this parameter, which was almost $60 \%$ higher than those attained when using corn cobs or cork oak as nutrient source. Such difference was due to the faster FOS production in this medium, which was maximum after only $16 \mathrm{~h}$ of fermentation. This productivity value $(8.05 \mathrm{~g} / \mathrm{l} \mathrm{h})$ represents also an important improvement in our previous result $(6.61 \mathrm{~g} / \mathrm{lh})$ achieved during the FOS production by SmF with immobilized A. japonicus [11]. The yield and productivity increase by using SSF systems instead of SmF systems has also been observed during the production of other compounds such as aromas, giberellic acid, ergot alkaloids, and others [12].

Fig. 2 shows the activity of the FFase enzyme during the FOS production from non-supplemented media. Note that the highest enzyme activity values were obtained in the medium containing coffee silverskin, which is in agreement with the elevated production of FOS observed in this medium. The lowest FFase results obtained in the media containing corn cobs or cork oak could be related to the presence of a lower nutrient amount in these two residues, when compared to coffee silverskin. According to Maiorano et al. [7], the properties of microbial FFase can vary according to the culture medium composition. The nitrogen concentration in the medium, for example, is reported as having influence in the enzyme production $[13,14]$.

Curiously, the medium containing synthetic fiber, which did not have FOS production when not supplemented with nutrients (Fig. 1B), gave considerable values of FFase activity. An explanation for this is that FFases commonly possess both transfructosylating (Ut) and hydrolytic (Uh) activities, which can be altered by modifying the reaction conditions [15]. For an efficient production of FOS, it is necessary to have a high Ut/Uh, ratio [16]. In this case, the activity observed for the assay with synthetic fiber was probably all of hydrolysis. In fact, chromatographic analysis of this fermentation medium revealed the presence of glucose and fructose in the medium besides sucrose, confirming the hydrolysis activity of the enzyme.

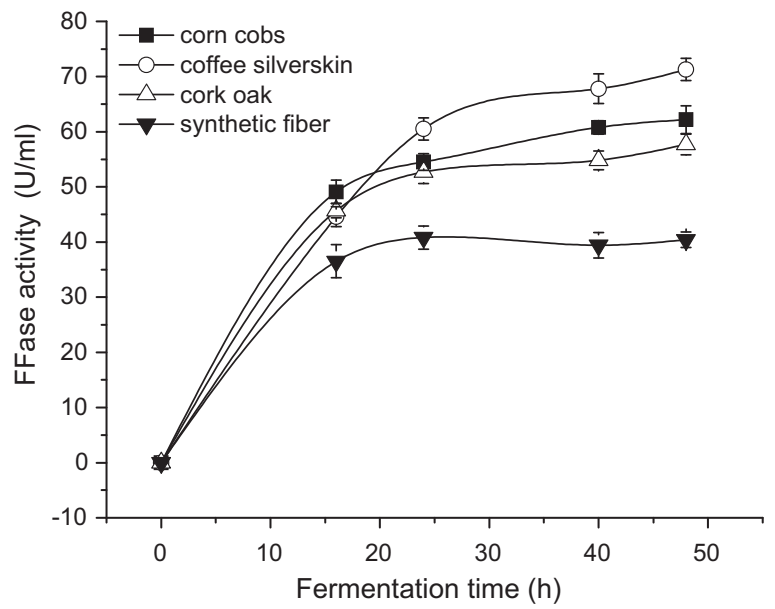

Fig. 2. $\beta$-Fructofuranosidase (FFase) activity during the FOS production by Aspergillus japonicus under solid-state fermentation conditions using different materials as solid support. Assays not supplemented with nutrients. 
It is worth mentioning the elevated values of FFase activity obtained in the present study. The highest value, $71.3 \mathrm{U} / \mathrm{ml}$, was obtained after $48 \mathrm{~h}$ fermentation in the medium using coffee silverskin as solid support and nutrient source. This value is favorably comparable to others reported in the literature with other microorganism strains. For example, $\mathrm{SmF}$ with $A$. japonicus JN-19 yielded a FFase activity of $55.42 \mathrm{U} / \mathrm{ml}$ after $96 \mathrm{~h}$ fermentation [17], and a mutant Saccharomyces cerevisiae produced FFase with activity of $45.65 \mathrm{U} / \mathrm{ml}$, after $48 \mathrm{~h}$ incubation time [18]. During the FOS production by $\mathrm{SmF}$ using $A$. japonicus immobilized in corn cobs, the maximum FFase activity value was $44.81 \mathrm{U} / \mathrm{ml}$ [11]. In the present work, SSF using corn cobs yielded a FFase activity of $62.2 \mathrm{U} / \mathrm{ml}$, demonstrating that this system improved the results previously obtained in SmF with immobilized cells. Other authors have also considered SSF more advantageous than SmF for the enzymes production, due to the higher fermentation productivity and concentrated product formation, besides to be of simpler operation, and less favorable for the contaminants growth [3].

Considering that the production and application of FFases has gained tremendous practical commercial importance due to their use on FOS production, the obtainment of this enzyme with high activity during the FOS production is a positive aspect for the development of a novel process with potential for synthesizing both products at the industrial level.

\section{Conclusion}

Corn cobs, coffee silverskin and cork oak can be used as nutrient source for FOS production by A. japonicus through SSF systems. Among these materials, coffee silverskin is able to provide all the nutritional requirements for the microorganism development since the results obtained when using this material as nutrient source were similar to those achieved when the medium was supplemented with nutrients. The highest enzymatic activity results were also achieved when using coffee silverskin as solid support. Such results are very promising and contribute to the establishment of a strategy for FOS production at industrial level with higher yields and productivities than those commonly achieved, and with lower operational costs due to the use of agro-industrial residues as nutrient source. In fact, SSF with coffee silverskin and A. japonicus can be considered an interesting strategy for synthesizing both products, FOS and FFase, at the industrial level.

\section{Acknowledgements}

This work was supported by grant SFRH/BPD/38212/2007 from the FCT (Portuguese Foundation for Science and Technology).
The authors thank NovaDelta-Comércio e Indústria de Cafés, S.A. (Campo Maior, Portugal) for the coffee silverskin donation.

\section{References}

[1] S.I. Mussatto, I.M. Mancilha, Non-digestible oligosaccharides: a review, Carbohydr. Polym. 68 (2007) 587-597.

[2] J.W. Yun, Fructooligosaccharides-occurrence, preparation, and application, Enzyme Microb. Technol. 19 (1996) 107-117.

[3] A.K. Balasubramaniem, K.V. Nagarajan, G. Paramasamy, Optimization of media for beta-fructofuranosidase production by Aspergillus niger in submerged and solid state fermentation, Process Biochem. 36 (2001) 12411247.

[4] C.-S. Chien, W.-C. Lee, T.-J. Lin, Immobilization of Aspergillus japonicus by entrapping cells in gluten for production of fructooligosaccharides, Enzyme Microb. Technol. 29 (2001) 252-257.

[5] S.I. Mussatto, L.R. Rodrigues, J.A. Teixeira, $\beta$-Fructofuranosidase production by repeated batch fermentation with immobilized Aspergillus japonicus, J. Ind. Microbiol. Biotechnol. 36 (2009) 923-928.

[6] K. Nishizawa, M. Nakajima, H. Nabetani, Kinetic study on transfructosylation by $\beta$-fructofuranosidase from Aspergillus niger ATCC 20611 and availability of a membrane reactor for fructooligosaccharide production, Food Sci. Technol. Res. 7 (2001) 39-44.

[7] A.E. Maiorano, R.M. Piccoli, E.S. Silva, M.F.A. Rodrigues, Microbial production of fructosyltransferases for synthesis of pre-biotics, Biotechnol. Lett. 30 (2008) 1867-1877.

[8] U. Hölker, J. Lenz, Solid-state fermentation: are there any biotechnological advantages? Curr. Opin. Microbiol. 8 (2005) 301-306.

[9] K.S.M.S. Raghavarao, T.V. Ranganathan, N.G. Karanth, Some engineering aspects of solid-state fermentation, Biochem. Eng. J. 13 (2003) 127-135.

[10] P.T. Sangeetha, M.N. Ramesh, S.G. Prapulla, Production of fructosyl transferase by Aspergillus oryzae CFR 202 in solid-state fermentation using agricultural byproducts, Appl. Microbiol. Biotechnol. 65 (2004) 530-537.

[11] S.I. Mussatto, C.N. Aguilar, L.R. Rodrigues, J.A. Teixeira, Fructooligosaccharides and $\beta$-fructofuranosidase production by Aspergillus japonicus immobilized on lignocellulosic materials, J. Mol. Catal. B-Enzym. 59 (2009) 76-81.

[12] U. Hölker, M. Höfer, J. Lenz, Biotechnological advances of laboratory-scale solidstate fermentation with fungi, Appl. Microbiol. Biotechnol. 64 (2004) 175186.

[13] I. Sarat Babu, S. Ramappa, D. Guru Mahesh, K. Sunanda Kumari, K. Sita Kumari, G. Subba, Optimization of medium constituents for the production of fructosyltransferase (FTase) by Bacillus subtilis using response surface methodology, Res. J. Microbiol. 3 (2008) 114-121.

[14] C. Dorta, R. Cruz, P. Oliva-Neto, D.J.C. Moura, Sugarcane molasses and yeast powder used in the fructooligosaccharides production by Aspergillus japonicusFCL 119T and Aspergillus niger ATCC 20611, J. Ind. Microbiol. Biotechnol. 33 (2006) 1003-1009.

[15] R.C. Fernández, B.G. Maresma, A. Juárez, J. Martínez, Production of fructooligosaccharides by $\beta$-fructofuranosidase from Aspergillus sp $27 \mathrm{H}$, J. Chem. Technol. Biotechnol. 79 (2004) 268-272.

[16] W.-C. Chen, C.-H. Liu, Production of $\beta$-fructofuranosidase by Aspergillus japonicus, Enzyme Microb. Technol. 18 (1996) 153-160.

[17] L.-M. Wang, H.-M.Zhou, Isolation and identification of a novel Aspergillus japonicus JN19 producing $\beta$-fructofuranosidase and characterization of the enzyme, J. Food Biochem. 30 (2006) 641-658.

[18] I. ul-Haq, S. Ali, A. Aslam, M.A. Qadeer, Characterization of a Saccharomyces cerevisiae mutant with enhanced production of $\beta$-D-fructofuranosidase, Bioresour. Technol. 99 (2008) 7-12. 